\title{
O Ácido (8)17,12E,14-labdatrieno-18-óico (labdano302), diterpeno tipo labdano isolado de Xylopia langsdorffiana St. Hil. \& Tul. (Annonaceae) relaxa a traquéia isolada de cobaia
}

\author{
Luciano A. A. Ribeiro ${ }^{1 *}$, Josean F. Tavares ${ }^{1}$, Nilza C. de Andrade ${ }^{2}$, Marcelo S. da Silva ${ }^{1}$, \\ Bagnólia A. da Silva ${ }^{1 *}$ \\ ${ }^{1}$ Laboratório de Tecnologia Farmacêutica "Prof. Delby Fernandes de Medeiros”, Universidade Federal da \\ Paraíba, Cx. Postal 5009, 58051-970, João Pessoa, Brasil, \\ ${ }^{2}$ Departamento de Química, Universidade Federal do Piauí, 64049-550, Teresina, PI, Brasil
}

\begin{abstract}
RESUMO: A espécie Xylopia langsdorffiana St. Hil.. \& Tul. é popularmente conhecida como "pimenteira-da-terra” no Sudeste do Brasil. A partir do fracionamento do extrato etanólico, obtido das cascas do caule desta espécie, foi isolado um diterpeno tipo labdano, identificado como sendo o ácido 8(17),12E,14-labdatrieno-18-óico, e que neste trabalho é codificado como labdano302. O labdano302 relaxou o tônus basal dos anéis de traquéia isolada de cobaia com um valor de $\mathrm{CE}_{50}$ de 6,7 $\pm 0,5 \times 10^{-8} \mathrm{M}$. O diterpeno labdano302 relaxou de maneira dependente de concentração os anéis pré-contraídos com carbacol $\left(10^{-6} \mathrm{M}\right)$, tanto na presença $\left(\mathrm{CE}_{50}=1,4 \pm 0,7 \times 10^{-5} \mathrm{M}\right)$ como na ausência de epitélio funcional $\left(\mathrm{CE}_{50}=1,5 \pm 0,3 \times 10^{-5} \mathrm{M}\right)$, bem como anéis pré-contraídos com 18 ou $60 \mathrm{mM}$ de $\mathrm{KCl}$, apresentando valores de $\mathrm{CE}_{50}$ de $2,3 \pm 0,4 \times 10^{-7} \mathrm{M}$ e 1,8 $\pm 0,8 \times 10^{-5} \mathrm{M}$, respectivamente. Este efeito relaxante, sobre as contrações induzidas por $18 \mathrm{mM}$ de $\mathrm{KCl}$, tanto foi significantemente mais potente quanto mais eficaz quando comparado ao efeito sobre as contrações induzidas por $60 \mathrm{mM}$ de $\mathrm{KCl}$. Assim, labdano302 mostra um efeito relaxante em traquéia isolada de cobaia, tanto em seu tônus basal como sob estímulo contrátil, aparentemente sem a participação dos fatores relaxantes derivados do epitélio, contudo com possível participação dos canais de $\mathrm{K}^{+}$.
\end{abstract}

Unitermos: Xylopia langsdorffiana, Annonaceae, diterpeno labdano, traquéia de cobaia, relaxamento.

\begin{abstract}
The (8)17,12E,14-labdatrien-18-oic acid (labdane302), labdane-type diterpene isolated from Xylopia langsdorffiana St. Hil. \& Tul. (Annonaceae) relaxes the guinea-pig trachea”. Xylopia langsdorfiana St. Hil. \& Tul. is popularly known as "pimenteira-da-terra” in Southeast of Brazil. The fractionation of the ethanol extract obtained from the stem-bark of this species yielded a labdane-type diterpene identified as 8(17),12E,14-labdatrien-18-oic acid, referred here as labdane302. In this study, we investigated the effect of labdane302 in guinea-pig trachea. labdane302 relaxed the basal tonus of trachea rings with $\mathrm{EC}_{50}$ value of $6.7 \pm 0.5 \times 10^{-8} \mathrm{M}$. The diterpene labdane302 relaxed the pre-contracted rings by carbachol $10^{-6} \mathrm{M}$ both in the presence $\left(\mathrm{EC}_{50}=1.4 \pm 0.7 \times 10^{-5} \mathrm{M}\right)$ and absence of functional epithelium $\left(\mathrm{EC}_{50}=1.5 \pm 0.3 \times 10^{-5} \mathrm{M}\right)$, as well as pre-contracted by $\mathrm{KCl} 18 \mathrm{mM}$ or $60 \mathrm{mM}$, presented $\mathrm{EC}_{50}$ values of $2.3 \pm 0.4 \times 10^{-7} \mathrm{M}$ and 1.8 $\pm 0.8 \times 10^{-5} \mathrm{M}$, respectively. This relaxant effect, upon contractions induced by $\mathrm{KCl} 18 \mathrm{mM}$, was more potent as well as more efficient than the one presented with $\mathrm{KCl} 60 \mathrm{mM}$ pre-contracted rings. The labdane-type diterpene labdane302 shows the relaxant effect in isolated guinea-pig trachea, pre-contracted or upon basal tonus, apparently without participation of epithelium-derived relaxant factors, but apparently involving activation of $\mathrm{K}^{+}$channels.
\end{abstract}

Keywords: Xylopia langsdorffiana, Annonaceae, labdane, diterpene, guinea-pig trachea, relaxation.

\section{INTRODUÇÃO}

A família Annonaceae é uma das maiores da classe Magnoliid possuindo 128 gêneros e aproximadamente 2300 espécies com distribuição pantropical (Kessler, 1993). No Brasil, a Annonaceae possui 26 gêneros e 260 espécies (Maas et al., 2001), dentre os quais se inclui o gênero Xylopia que possui aproximadamente 160 espécies (Takahashi et al., 2001). Vários produtos naturais pertencentes a diversas classes químicas têm sido isolados de plantas do gênero Xylopia, incluindo acetogeninas (Alfonso et al., 1996), alcalóides (Martins et al., 1998); flavonóides (Santos; Salatino, 2000), sesquiterpenos (Moreira et al., 2007) e diterpenos tipo labdano e caurano (Vilegas et al., 1991; Martins et al., 1999; Melo et al., 2001; Andrade 
et al., 2004). Os diterpenos são substâncias comuns às espécies de Xylopia e acredita-se que essa classe de compostos é característica do gênero Xylopia (Vilegas et al., 1991), tornando-os potencialmente importantes como marcadores quimiotaxonômicos de espécies pertencentes a esse gênero.

A espécie Xylopia langsdorffiana St. Hil. \& Tul. é uma árvore medindo entre 5 e 7 metros de altura (Maas et al., 2001), e é popularmente conhecida como “pimenteira-da-terra” no Sudeste do Brasil (Corrêa, 1984). Em trabalhos anteriores foram isolados diterpenos do tipo trachilobano (Tavares et al., 2006) e atisano (Tavares et al; 2007). A partir do extrato etanólico bruto, obtido das cascas do caule dessa espécie, também foi isolado um diterpeno tipo labdano, sendo este identificado por comparação de seus dados de ponto de fusão (139-141 ${ }^{\circ} \mathrm{C}$ ), espectroscopia de ressonância nuclear magnética de hidrogênio e carbono ( $\mathrm{RMN}$ de ${ }^{1} \mathrm{H}$ e ${ }^{13} \mathrm{C}$ ) com dados previamente publicados por Bohlmann et al. (1980), como sendo o ácido 8(17),12E,14-labdatrieno-18-óico (Figura 1), com fórmula molecular $\mathrm{C}_{20} \mathrm{H}_{30} \mathrm{O}_{2}$ e massa molecular de 302,451 u.m.a. (Tavares et al., 2007), neste trabalho codificado por labdano302.

Alguns diterpenos tipo labdano têm demonstrado ações farmacológicas em músculos lisos, como por exemplo, atividade antiespasmódica de 1,9dideoxiforscolina (Vedernikov et al., 2000) e de jatrofone em útero isolado de rata (Calixto; Sant'Ana, 1990); atividade anti-hipertensiva de 13-epi-9-deoxiforscolina em ratos (Tandon et al., 1992); atividade relaxante de jatrofone em aorta isolada de rato (Duarte et al., 1992). Em um trabalho recentemente publicado, Oliveira et al. (2006) mostraram que o diterpeno labdano302 possui tanto efeito hipotensor, como vasorelaxante em artéria mesentérica superior, em ratos normotensos.

Assim, o objetivo deste trabalho foi estudar os efeitos relaxantes do labdano302 sobre a traquéia isolada de cobaia, usando para tanto uma abordagem farmacológica, visando contribuir para o estudo de produtos naturais com possível potencial terapêutico.

\section{MATERIAL E MÉTODOS}

\section{Material vegetal}

O caule de Xylopia langsdorffiana foi coletado no município de Cruz do Espírito Santo, estado da Paraíba, em julho de 2002. O material botânico foi identificado pela Profa. Dra. Maria de Fátima Agra, do Setor de Botânica do LTF/UFPB, e uma exsicata da planta está depositada no Herbário Prof. Lauro Pires Xavier (JPB), da UFPB, sob identificação AGRA 5541.

\section{Substâncias}

O sulfato de magnésio hepta-hidratado $\left(\mathrm{MgSO}_{4} \cdot 7 \mathrm{H}_{2} \mathrm{O}\right)$, bicarbonato de sódio $\left(\mathrm{NaHCO}_{3}\right)$, cloreto de cálcio bi-hidratado $\left(\mathrm{CaCl}_{2} \cdot 2 \mathrm{H}_{2} \mathrm{O}\right)$, cloreto de sódio $(\mathrm{NaCl})$, fosfato ácido de potássio mono-hidrato $\left(\mathrm{KH}_{2} \mathrm{PO}_{4}\right.$. $\mathrm{H}_{2} \mathrm{O}$ ), glicose; cloridrato de carbamilcolina (carbacol) e o cloreto de potássio $(\mathrm{KCl})$ foram obtidos da Merck (Brasil). O cloridrato de isoproterenol (isoprenalina), o ácido araquidônico, cremofor e o ácido clorídrico $(\mathrm{HCl})$ foram obtidos da Sigma-Aldrich (USA). O etanol foi obtido da Reagen (Brasil).

Todas as substâncias foram dissolvidas em água, exceto o ácido araquidônico que foi dissolvido em etanol PA (95 \%). O labdano302 foi dissolvido em cremofor e diluído em água destilada até a concentração de $10^{-2} \mathrm{M}$ (a concentração de cremofor na solução foi de 3 \% v/v). A concentração de cremofor na cuba nunca ultrapassou 0,01 \% (v/v) nos experimentos realizados, sendo assim desprovido de efeitos contráteis ou relaxantes no órgão em estudo.

\section{Preparação do tecido}

Os experimentos foram realizados com anéis de traquéia isolada de cobaias de ambos os sexos (300-400 g). Os animais foram sacrificados por concussão cerebral e em seguida exsanguinados, seguindo os princípios de cuidados com animais aprovados pelo Comitê de Ética em Pesquisa Animal do LTF/UFPB (Parecer CEPA $n^{\circ}$ 0511/05). A traquéia foi imediatamente removida e imersa em solução de Krebs normal e borbulhada continuamente com mistura carbogênica (95\% de $\mathrm{O}_{2}$ e $5 \%$ de $\mathrm{CO}_{2}$ ). A composição (em mM) da solução de Krebs utilizada foi: $\mathrm{NaCl}(118) ; \mathrm{KCl}(4,6) ; \mathrm{MgSO}_{4} .7 \mathrm{H}_{2} \mathrm{O}(5,7) ; \mathrm{KH}_{2} \mathrm{PO}_{4}$. $\mathrm{H}_{2} \mathrm{O}(1,1) ; \mathrm{CaCl}_{2} \cdot \mathrm{H}_{2} \mathrm{O}(2,5) ; \mathrm{NaHCO}_{3}$ (25); glicose (11). $\mathrm{O}$ valor de $\mathrm{pH}$ foi ajustado para 7,4 com solução de $\mathrm{HCl}$ a $1 \mathrm{~N}$. A traquéia foi cortada em segmentos de 3-4 anéis de cartilagem que foram montados entre duas hastes de aço inox, uma presa à cuba de vidro e a outra a um transdutor de força (FORT10) conectado a um amplificador (Transbridge 4M), ambos da World Precision Instruments Inc. (Sarasota, USA), que estavam conectados a uma placa conversora A/D instalada em um computador usando o programa BioMed (Biodata, Brasil). As preparações foram imersas em cubas de vidro para órgão isolados de $6 \mathrm{~mL}$ contendo solução de Krebs e aerada com mistura carbogênica à $37^{\circ} \mathrm{C}$. Os anéis de traquéia foram deixados em repouso por um período de 60 min para estabilização da preparação a uma tensão de 1 g.

\section{Efeito de labdano302 sobre o tônus basal dos anéis de traquéia isolada de cobaia}

Após o período de estabilização, dois relaxamentos foram induzidos pela adição de $10^{-6} \mathrm{M}$ de isoprenalina à cuba, e a suas amplitudes máximas foram consideradas como controle (100\%). Após um intervalo não menor que $30 \mathrm{~min}$, suficiente para o retorno do órgão a seu tônus basal normal, o labdano302 foi adicionado cumulativamente à cuba e seu efeito foi expresso como 
a porcentagem do relaxamento máximo induzido pela isoprenalina. Os anéis de traquéia de cobaia usados nestes experimentos não foram submetidos a nenhum processo mecânico para a retirada da camada epitelial, sendo assim considerados como íntegros. Contudo, o grau de integridade desse epitélio não foi determinado experimentalmente.

Efeito de labdano302 sobre anéis de traquéia précontraídos com carbacol na presença e na ausência de epitélio funcional

Em um outro conjunto de experimentos foi avaliado o efeito de labdano302 sobre os anéis de traquéia pré-contraídos com carbacol (CCh), na presença ou na ausência do epitélio funcional. Após o período de estabilização, duas contrações de magnitude similares foram induzidas com $10^{-6} \mathrm{M}$ de $\mathrm{CCh}$ e consideradas como controle. A integridade do epitélio da traquéia foi verificada pela adição de ácido araquidônico $\left(10^{-4}\right.$ M) à cuba durante a fase tônica da primeira contração induzida por CCh (Tschirhart et al., 1987). Os anéis de traquéia que exibiam relaxamentos superiores a 50\% (em relação à força de contração inicial) foram considerados com epitélio funcional, caso não houvesse relaxamento ou esse fosse inferior a $10 \%$ o anel foi considerado sem epitélio. Os anéis de traquéia sem epitélio foram obtidos através da retirada do mesmo por atrito entre a parede interna do órgão e uma haste envolta em algodão e embebida com solução de Krebs. Durante a fase tônica da segunda contração, o labdano302 foi adicionado de maneira cumulativa à cuba. $\mathrm{O}$ relaxamento foi expresso como a percentagem reversa da força de contração inicial máxima induzida por CCh.

\section{Efeito de labdano302 sobre os anéis de traquéia pré-} contraídos com 18 ou $60 \mathrm{mM}$ de $\mathrm{KCl}$

Foi avaliado o efeito relaxante de labdano302 sobre as contrações tônicas induzidas por aumento da concentração de $\mathrm{K}^{+}$na solução de Krebs. Duas soluções foram usadas, solução de Krebs com $18 \mathrm{mM}$ de $\mathrm{K}^{+}$ (KCl 18) ou solução de Krebs com $60 \mathrm{mM}$ de $\mathrm{K}^{+}$(KCl 60 ), onde houve a troca equimolar de $\mathrm{NaCl}$ por $\mathrm{KCl}$ no preparo da solução de Krebs normal. Após o período de estabilização e teste da integridade ou não do epitélio da traquéia, duas contrações de magnitude similares foram induzidas pela troca de solução de Krebs por solução de KCl 18 ou 60 mM e consideradas como controle. Durante a fase tônica da segunda contração induzida por $\mathrm{KCl}$, o labdano302 foi adicionado de maneira cumulativa à cuba. O relaxamento foi expresso como a percentagem reversa da força de contração inicial máxima induzida por $\mathrm{KCl}$. Nestes experimentos, só foram utilizados os anéis de traquéia sem epitélio funcional.

\section{Analise estatística}

Os resultados obtidos foram analisados estatisticamente empregando-se o teste $t$ de Student (não-pareado), onde essas diferenças foram consideradas estatisticamente significantes quando os valores de $p$ foram menores que 0,05 .

Os valores de $\mathrm{CE}_{50}$ (concentração de uma substância que produz uma resposta de $50 \%$ de seu efeito máximo) (Neubig et al., 2003) foram calculados por regressão não-linear (usando a equação de Hill) e estão apresentados como média \pm erro padrão da média (média \pm e.p.m.) em todos os experimentos realizados. Todos os dados foram analisados pelo programa GraphPad Prism ${ }^{\odot}$ versão 4.03 (GraphPad Software Inc., USA).

\section{RESULTADOS}

Efeito de labdano302 sobre o tônus basal dos anéis de traquéia isolada de cobaia

O labdano302 (10-9 - 10-4 M) foi capaz de relaxar o tônus basal da traquéia isolada de cobaia de maneira dependente de concentração, atingindo um efeito máximo $\left(\mathrm{E}_{\max }\right)$ de $100 \%$, em relação ao relaxamento máximo produzido por $10^{-6} \mathrm{M}$ de isoprenlina. Sobre o tônus espontâneo o $\mathrm{E}_{\max }$ de labdano302 foi alcançado entre as concentrações de $10^{-5}$ e $3 \times 10^{-5} \mathrm{M}$, e o valor de $\mathrm{CE}_{50}$ apresentando foi de 6,7 \pm 0,5 x 10-8 M (Gráfico $1 \mathrm{~A}$ ). O tempo médio para que o labdano302 produzisse o seu $\mathrm{E}_{\max }$ foi de $150 \pm 11 \mathrm{~min}$.

Efeito de labdano302 sobre anéis de traquéia précontraídos na presença e na ausência de epitélio funcional

O diterpeno labdano302 $\left(10^{-9}\right.$ a $\left.10^{-4} \mathrm{M}\right)$ também relaxou os anéis de traquéia isolada de cobaia précontraídos com $10^{-6} \mathrm{M}$ de CCh, de maneira dependente de concentração, tanto na presença $\left(\mathrm{CE}_{50}=1,4 \pm 0,7 \times 10^{-5} \mathrm{M}\right)$ como na ausência $\left(\mathrm{CE}_{50}=1,5 \pm 0,3 \times 10^{-5} \mathrm{M}\right)$ de epitélio funcional $(n=5)$, não havendo diferença estatística entre os seus valores de $\mathrm{CE}_{50}$. $\mathrm{O} \mathrm{E}_{\max }, 100 \%$ de relaxamento, foi alcançado na concentração de $10^{-4} \mathrm{M}$ independentemente da presença ou não de epitélio funcional (Gráfico 2). O tempo médio para que o labdano302 alcançasse seu $\mathrm{E}_{\max }$ foi de $215 \pm 24$ min e $183 \pm 32 \mathrm{~min}$, para anéis com e sem epitélio funcional, respectivamente.

\section{Efeito de labdano302 sobre os anéis de traquéia pré- contraídos com 18 e $60 \mathrm{mM}$ de $\mathrm{KCl}$}

O labdano302 relaxou de maneira dependente de concentração $\left(10^{-8}-10^{-4} \mathrm{M}\right)$ os anéis de traquéia pré-contraídos com 18 e $60 \mathrm{mM}$ de $\mathrm{KCl}$, apresentando valores de $\mathrm{CE}_{50}$ de $2,3 \pm 0,4 \times 10^{-7} \mathrm{M}$ e $1,8 \pm 0,8 \times 10^{-}$ ${ }^{5} \mathrm{M}$, respectivamente, que se mostram estatisticamente diferentes. $\mathrm{O}$ valor de $\mathrm{E}_{\max }$ do labdano302 foi de $100 \%$, 
quando as contrações foram induzidas com $18 \mathrm{mM}$ de $\mathrm{KCl}$, sendo alcançado na concentração de $10^{-5} \mathrm{M}$. Entretanto, quando as contrações foram induzidas por 60 mM de $\mathrm{KCl}$ o labdano302 teve um $\mathrm{E}_{\text {max }}$ médio de 74,6\%, sendo significantemente menor que o obtido na contração induzida com $18 \mathrm{mM}$ de $\mathrm{KCl}$, e somente foi alcançado numa concentração dez vezes maior (Gráfico 2). O tempo médio para que o labdano302 alcançasse seu $\mathrm{E}_{\max }$ foi de $90 \pm 11$ e $135 \pm 15$ min sobre a traquéia de cobaia précontraída por 18 e $60 \mathrm{mM}$ de $\mathrm{KCl}$, respectivamente.

Em todos os resultados os efeitos do labdano302 foram reversíveis após 30 minutos após a troca das soluções contidas nas cubas, e assim sua retirada das cubas.

\section{DISCUSSÃO}

No presente estudo demonstra-se, pela primeira vez, evidências farmacológicas de que o ácido 8(17),12E,14-labdatrieno-18-óico (labdano302), isolado da espécie Xylopia langsdorffiana St. Hil. \& Tul. (Tavares et al., 2007), apresenta efeito relaxante em traquéia isolada de cobaia por um mecanismo aparentemente independente de fatores relaxantes derivados do epitélio, porém dependente da ativação de canais de $\mathrm{K}^{+}$.

O tônus miogênico das vias aéreas é mantido devido ao influxo passivo de íons $\mathrm{Ca}^{2+}$ através de vários canais de membrana, que proporciona uma quantidade suficiente para manter os níveis basais de $\mathrm{Ca}^{2+}$ intracelulares e a quantidade desse íon armazenada no retículo sarcoplasmático, assim como esse influxo ajuda a manter o potencial de membrana dessas células (Montano; Bazan-Perkins, 2005). Nas células do músculo liso da traquéia, o subtipo de $\beta$-adrenoceptor mais expresso é o receptor $\beta_{2}$, que possui um papel importante na manutenção do tônus neste órgão. $\mathrm{O}$ efeito final decorrente da ativação de receptores $\beta_{2}$ depende da ativação da ciclase de adenilil (AC) e conseqüente aumento nos níveis de 3',5'-monofosfato de adenosina cíclico (AMPc), sendo esse o principal segundo mensageiro responsável pela a modulação da contratilidade neste músculo (Tanaka; Horinouchi; Koibe, 2005). Neste trabalho foi demonstrado que o diterpeno labdano302 é capaz de relaxar a traquéia em seu tônus basal (Gráfico $1 \mathrm{~A})$, sem que nenhum estímulo seja aplicado a ela, além da tensão inicial. Este dado é sugestivo de que o labdano302 exerce seu efeito relaxante por interferir com os mecanismos responsáveis pela manutenção do tônus basal na traquéia, como por exemplo a modulação de canais de $\mathrm{K}^{+}$, como é relatado para a isoprenalina, um agonista dos $\beta$-adrenoceptores (Tanaka et al., 2005).

Há relatos de que a remoção mecânica do epitélio da traquéia aumenta a resposta a vários broncoconstritores (histamina, acetilcolina, leucotrienos, adenosina, substância $\mathrm{P}$ e endotelina) como também broncodilatadores (isoprenalina e nitroprussiato de sódio) (Fedan; Scott; Johnston, 2001). Baseando-se na propriedade do ácido araquidônico de produzir um relaxamento em traquéia de cobaia de maneira dependente de epitélio e bloqueado por indometacina e ácido acetilsalicílico (Pelaia et al., 2002), esse foi usado para determinar a integridade do epitélio, e assim investigar a participação dos fatores derivados do epitélio no efeito relaxante do labdano302 em traquéia pré-contraída com um análogo da acetilcolina, o CCh. No entanto, a presença ou ausência de epitélio funcional não modificou significantemente a curva concentração-resposta do labdano302 na traquéia isolada de cobaia. $\mathrm{O}$ diterpeno relaxou os anéis com e sem epitélio com a mesma potência e atingindo o mesmo efeito máximo (Gráfico $1 \mathrm{~B}$ ), o que sugere que os fatores relaxantes derivados do epitélio provavelmente não estão envolvidos nesse efeito relaxante. Além disso, observou-se que o efeito relaxante do labdano302 não é alterado pela presença da indometacina, um inibidor das ciclooxigenases, ou de propranolol, um antagonista dos $\beta$-adrenoceptores (dados não mostrados).

A contração muscular da traquéia é largamente determinada pela concentração de $\mathrm{Ca}^{2+}$ intracelular $\left[\mathrm{Ca}^{2+}\right]_{i}$. A contração deflagrada por broncoconstritores, como por exemplo, a acetilcolina e o CCh, resulta num aumento na $\left[\mathrm{Ca}^{2+}\right]_{\mathrm{i}}$ de $0,1 \mu \mathrm{M}$ para $1 \mu \mathrm{M}$, sendo esse aumento de 10 vezes nos níveis de $\mathrm{Ca}^{2+}$ responsável por ativar a maquinaria contrátil gerando o aumento no tônus da traquéia (Barnes, 1998). A contração do músculo liso é, em última instância, dependente do aumento da $\left[\mathrm{Ca}^{2+}\right]_{\text {i }}$. Enquanto a liberação de $\mathrm{Ca}^{2+}$ dos estoques intracelulares, mediado por ativação por agonistas, contribui para início do desenvolvimento da força de contração (Somlyo; Somlyo, 1994), a manutenção desta contração depende absolutamente do influxo sustentado de $\mathrm{Ca}^{2+}$ através de canais na membrana plasmática abertos por variação no potencial de membrana (Huang, 1998). Além disso, sabe-se que o epitélio da traquéia possui um papel importante em modular as respostas a vários agonistas no músculo liso das vias aéreas. Neste contexto, os canais de potássio desempenham um papel chave na regulação do potencial de membrana e na excitabilidade celular, sendo a contração no músculo liso depende do balanço entre o aumento da condutância ao íon $\mathrm{K}^{+}$, levando a uma hiperpolarização, e a diminuição da condutância ao $\mathrm{K}^{+}$, levando a uma despolarização (Knot et al., 1996). Esse balanço entre hiperpolarização e despolarização determina principalmente o influxo de $\mathrm{Ca}^{2+}$ através dos anais de $\mathrm{Ca}^{2+}$ abertos por voltagem $\left(\mathrm{Ca}_{\mathrm{v}}\right)$ (Huang, 1998). Os agonistas dos receptores $\beta_{2}$, como a isoprenalina, produzem hiperpolarização de membrana em traquéia de cobaia, e os principais canais de $\mathrm{K}^{+}$envolvidos neste efeito são canais de $\mathrm{K}^{+}$de grande condutância ativados pelo $\mathrm{Ca}^{2+}\left(\mathrm{BK}_{\mathrm{Ca}}\right)$, os canais de $\mathrm{K}^{+}$sensíveis ao ATP $\left(\mathrm{K}_{\mathrm{ATP}}\right)$ e os canais de $\mathrm{K}^{+}$ativados por voltagem $\left(\mathrm{K}_{\mathrm{v}}\right)$, sendo que todos estes canais são modulados positivamente por fosforilação via proteína cinase dependente de AMPc, a PKA (Tanaka et al., 2005). 
Para verificar se o labdano302 estaria agindo por diminuir o influxo de $\mathrm{Ca}^{2+}$ através do bloqueio direto dos $\mathrm{Ca}_{\mathrm{v}}$ ou por um bloqueio indireto, através da abertura de canais de $\mathrm{K}^{+}$, avaliou-se o efeito relaxante desse diterpeno sobre as contrações induzidas por aumento moderado das concentrações de $\mathrm{K}^{+}(\mathrm{KCl} 18 \mathrm{mM})$ e por altas concentrações de $\mathrm{K}^{+}(\mathrm{KCl} 60 \mathrm{mM})$. Sabese que ativadores de canais de $\mathrm{K}^{+}$, como por exemplo cromacalina e pinacidil, são mais efecientes em relaxar o músculo liso pré-contraído em resposta a um aumento moderado de $\mathrm{K}^{+}$extracelular, como é o caso de $\mathrm{KCl} 18$ (mM), do que em resposta a um aumento acentuado, como na situação de $\mathrm{KCl} 60$ (mM) (para revisão ver Gurney, 1994 e Underwood; Raeburn, 1995). Os resultados do presente estudo mostram que o labdano302 é capaz de relaxar com maior potência e eficácia a traquéia de cobaia pré-contraída com $18 \mathrm{mM}$ do que com $60 \mathrm{mM}$ de $\mathrm{KCl}$ (Gráfico 2). Essa diferença na ação do labdano302 sugere que o efeito relaxante desse diterpeno seja devido a uma abertura de canais de $\mathrm{K}^{+}$, uma vez que um aumento na

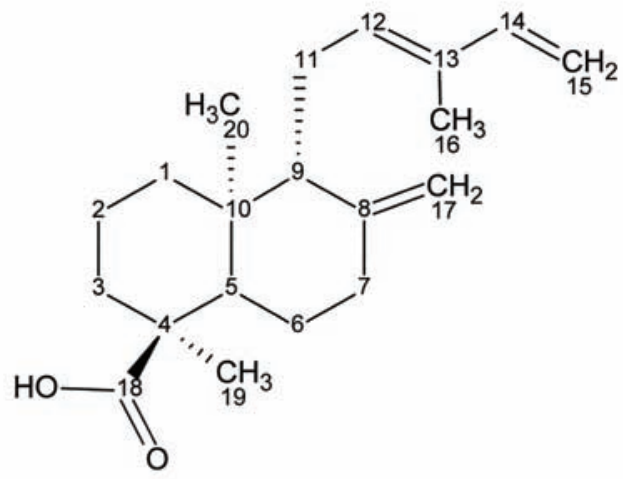

Figura 1. Estrutura química do ácido 8(17),12E,14-labdatrieno-18-óico (labdano302).
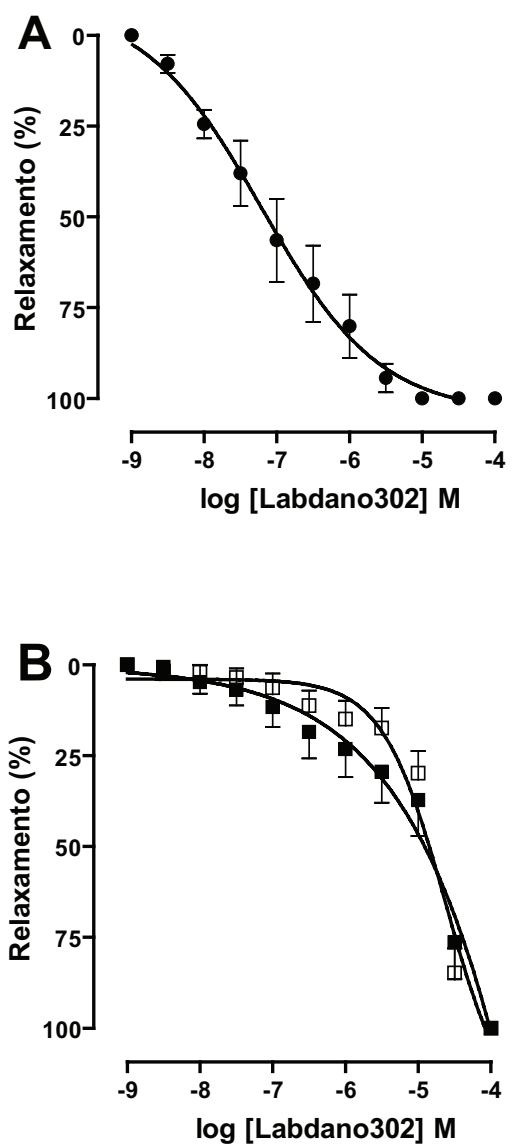

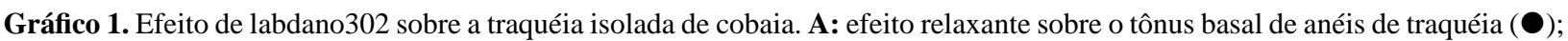
B: efeito relaxante sobre os anéis pré-contraídos com $1 \mu \mathrm{M}$ de carbacol, na presença ( $\square$ ) e na ausência ( $\square$ ) de epitélio funcional. Os símbolos e as barras representam a média e o e.p.m., respectivamente $(\mathrm{n}=5)$. 


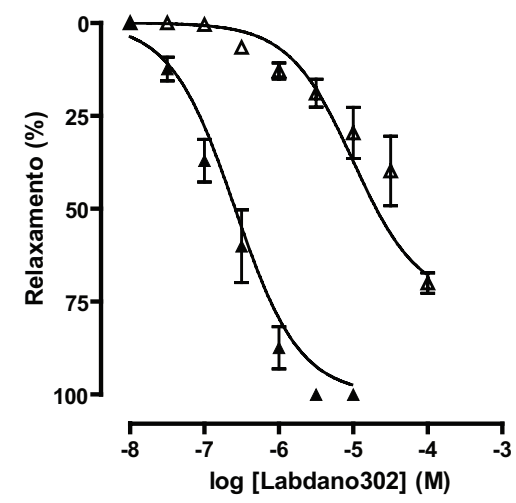

Gráfico 2. Efeito relaxante de labdano302 sobre os anéis de traquéia isolada de cobaia pré-contraída com $18 \mathrm{mM}(\boldsymbol{\Delta}, \mathrm{n}=5)$ ou 60 $\mathrm{mM}(\triangle, \mathrm{n}=3)$ de $\mathrm{KCl}$. Os símbolos e as barras representam a média e o e.p.m., respectivamente.

concentração extracelular de $\mathrm{K}^{+}$promove uma redução no gradiente eletroquímico para o $\mathrm{K}^{+}$e em altas concentrações extracelulares desse íon o efeito de ativadores de canais de $\mathrm{K}^{+}$é sensivelmente reduzido (Nielsen-Kudsk, 1996).

Substâncias relaxantes que agem no sistema respiratório são de grande relevância, podendo ser potenciais agentes no tratamento de desordens relacionadas à contratilidade do músculo liso das vias aéreas, como por exemplo, a asma (Pelaia et al., 2002). Tomados em conjunto os resultados demonstram que o ácido 8(17),12E,14-labdatrieno-18-óico (labdano302) isolado da espécie Xylopia langsdorffiana St.-Hil. \& Tul. apresenta um efeito relaxante em traquéia isolada de cobaia, e que em nível funcional, este efeito aparentemente não depende de fatores relaxantes derivados do epitélio, porém parece depender da ativação de canais de $\mathrm{K}^{+}$.

\section{AGRADECIMENTOS}

Os autores agradecem ao Sr. José Crispim Duarte e Sr. Vicente Carlos de Oliveira Costa pela assistência técnica, à CAPES e ao CNPq pelo apoio financeiro.

\section{REFERÊNCIAS}

Alfonso D, Colman-Saizarbitoria T, Zhao GX, Shi G, Ye Q, Schwedler JT, Mclaughlin JL 1996. Aromin and aromicin, two new bioactive annonaceous acetogenins, prossessing an unusual bis-thf ring structure, from Xylopia aromatica (Annonaceae). Tetrahedron 52: 4215-4224.

Andrade NC, Barbosa-Filho JM, Silva MS, Cunha EVL, Maia JGS 2004. Diterpenes and volatile constituents from the leaves of Xylopia cayennensis Maas. Biochem Syst Ecol 32: 1055-1058.

Barnes PJ 1998. Pharmacology of airway smooth muscle. Am J Respir Crit Care Med 158: S123-S132.

Bohlmann F, Jakupovic J, King RM, Robinson H 1980. Neue ent-atisiren- und ent-kaurensaure-derivate aus helianthus-arten. Phytochemistry 19: 863-868.
Calixto JB, Sant'Ana AEG 1990. Evidence for the mechanism of the inhibitory action of jatrophone in the isolated rat uterine muscle. Gen Pharmacol 21: 117-122.

Corrêa PM 1984. Dicionário de plantas úteis do Brasil e exóticas cultivadas. Brasilia: IBDF Ministério da agricultura.

Duarte DFP, Sant'Ana AEG, Calixto JB 1992. Analysis of the vasorelaxant action of jatrophone in the isolated aorta of the rat: influence of potassium channel blockers. Eur J Pharmacol 215: 75-81.

Fedan JS, Van Scott MR, Johnston RA 2001. Pharmacological techniques for the in vitro study of airways. $J$ Pharmacol Toxicol Methods 45: 159-174.

Gurney AM 1994. Mechanisms of drug-induced vasodilation. $J$ Pharm Pharmacol 46: 242-251.

Huang Y 1998 Hydroxylamine-induced relaxation inhibited by $\mathrm{K}^{+}$channel blockers in aortic rings. Eur J Pharmacol 349: 53-60.

Kessler PJA 1993. Annonaceae.. In: Kubitzki, K. Rohwer, J.G. \&Bittrich, V. (org.). The families and genera of vascular plants. Vol. 2. Flowering plants. Dicotyledons. Magnoliid, Hamamelid and Caryophyllid families. Berlin: SpringerVerlag, p. 93-129.

Knot HT, Brayden EJ, Nelson MT, Bárány M 1996. Calcium channels and potassium channels. In: Bárány, M. Biochemistry of smooth muscle contraction. San Diego: Academic Press, p. 203-219.

Maas PJM, Kamer HM, Junikka L, Mello-Silva R, Rainer $\mathrm{H}$ 2001. Annonaceae from Central-eastern Brazil. Rodriguésia 52: 65-98.

Martins D, Hamarski L, Alvarenga SAV, Roque NF 1999. Labdane dimers from Xylopia aromatica. Phytochemistry 51: 813-817.

Martins D, Osshiro E, Roque NF, Marks V, Gottlieb HE 1998. A sesquiterpene dimer from Xylopia aromatica. Phytochemistry 48: 677-680.

Melo AC, Cota BB, Oliveira AB, Braga FC 2001. HPLC quantification of kaurene diterpenes in Xylopia species. Fitoterapia 72: 40-45.

Montano LM, Bazan-Perkins B 2005. Resting calcium influx in airway smooth muscle. Can J Physiol Pharmacol 


$$
\text { 83: 717-723. }
$$

MoreiraIC, RoqueNF, ContiniK,LagoJHG2007.Sesquiterpenos e hidrocarbonetos dos frutos de Xylopia emarginata (Annonaceae). Rev Bras Farmacogn 17: 55-58.

Neubig RR, Spedding M, Kenakin T, Christopoulos A 2003. International Union of Pharmacology Committee on Receptor Nomenclature and Drug Classification. XXXVIII. Update on Terms and Symbols in Quantitative Pharmacology. Pharm Rev 55: 597-606.

Nielsen-Kudsk JE 1996. Potassium channel modulation: a new drug principle for regulation of smooth muscle contractility. Studies on isolated airways and arteries. Dan Med Bull 43: 429-447.

Oliveira AP, Furtado FF, Silva MS, Tavares JF, Mafra RA, Araújo DAM, Cruz JS, Medeiros IA 2006. Calcium channel blockade as a target for the cardiovascular effects induced by the 8 (17),12E, 14-labdatrien-18oic acid (labdane-302). Vasc Pharmacol 44: 338-344.

Pelaia G, Gallelli L, Vatralla A, Grembiale RD, Maselli R, De Sarro GB, Marsico S 2002. A Potential role of potassium channel openers in the treatment of asthma and chronic obstructive pulmonary disease. Life Sci 70: 977-990.

Santos DYAC, Salatino MLF 2000. Foliar flavonoids of Annonaceae from Brazil: taxonomic significance. Phytochemistry 55: 567-573.

Somlyo AP, Somlyo AV 1994. Signal transduction and regulation in smooth muscle. Nature 372: 231-236.

Takahashi JA, Vieira HS, Boaventura MAD 2001. Mono and diterpenes from seeds of Xylopia sericea. Quim Nova 24: 616-618.

Tanaka Y, Yamashita Y, Horinouchi T, Koike K 2005. Adrenaline produces the relaxation of guinea-pig airway smooth muscle primarily through the mediation of $\beta 2$ adrenoceptors. J Smooth Muscle Res 41: 153-161.

Tanaka Y, Horinouchi T, Koike K 2005. New insights into $\beta$ -adrenoceptors in smooth muscle: distribution of receptor subtypes and molecular mechanisms triggering muscle relaxation. Clin Exp Pharmacol Physiol 32: 503-514.

Tandon JS, Roy R, Balachandran S, Vishwakarma RA 1992. Epi-deoxycoleonol, a new antihipertensive labdane diterpenoid from Coleus forskohlii. Bioorg Med Chem Lett 2: 249-254.

Tavares JF, Queiroga KF, Silva MVB, Diniz MFFM, BarbosaFilho JM, Cunha EVL, Simone CA, Araújo-Júnior JX, Melo PS, Haun M, Silva MS 2006. ent-Trachylobane diterpenoids from Xylopia langsdorffiana. J Nat Prod 69: 960-962.

Tavares JF, Silva MVB, Queiroga KF, Diniz MFFM, BarbosaFilho JM, Haun M, Melo PS, Silva MS 2007. Xylodiol, a new atisane diterpenoid from Xylopia langsdorffiana St.-Hil. \& Tul. (Annonaceae). Z Naturforsch 62b: 742-744.

Tschirhart E, Frossard N, Bertrand C, Landry Y 1987. Arachidonic acid metabolites and airway epitheliumdependent relaxant factor. J Pharm Exp Ther 243:
310-316.

Underwood SL, Raeburn D 1995. Adenosine triphosphateactivated potassium channels. In: Raeburn, D. and Giembycz, M.A.. Airways Smooth Muscle: Peptide Receptors, Ion channels and Signal Transduction. Switzerland: Birkhäuser Verlag Basel, p. 199-216.

Vedernikov YP, Syal AS, Okawa T, Saade GR, Garfield RE 2000. Adenylate cyclase and potassium channels are involved in forskolin and 1,9-dideoxyforskolin induced inhibition of pregnant rat uterus contractility. Am J Obstet Gynecol 182: 620-624.

Vilegas W, Felicio JD, Roque NF, Gottlieb HE 1991. Diterpenic adducts from Xylopia species. Phytochemistry 30: 1869-1872. 